\title{
Implementing Affect Parameters in Personalized Web-Based Design
}

\author{
Zacharias Lekkas ${ }^{1}$, Nikos Tsianos ${ }^{1}$, Panagiotis Germanakos ${ }^{2,3}$, Constantinos Mourlas ${ }^{1}$, \\ and George Samaras ${ }^{3}$ \\ ${ }^{1}$ Faculty of Communication and Media Studies, National \& Kapodistrian University of \\ Athens, 5 Stadiou Str, GR 105-62, Athens, Hellas \\ zlekkas@gmail.com, \{ntsianos, mourlas\}@media.uoa.gr \\ 2 Department of Management and MIS, University of Nicosia, 46 Makedonitissas Ave., \\ P.O. Box 24005, 1700 Nicosia, Cyprus \\ ${ }^{3}$ Computer Science Department, University of Cyprus, CY-1678 Nicosia, Cyprus \\ \{pgerman, cssamara\} @cs.ucy.ac.cy
}

\begin{abstract}
Researchers used to believe that emotional processes are beyond the scope of a scientific study. Recent advances in cognitive science and artificial intelligence, however, suggest that there is nothing mystical about emotional processes. Affective neuroscience and psychology have reported that human affect and emotional experience play a significant, and useful, role in human learning and decision making. Emotions are considered to play a central role in guiding and regulating learning, performance, behaviour and decision making, by modulating numerous cognitive and physiological activities. Our purpose is to improve learning performance and, most importantly, to personalize web-content to users' needs and preferences, eradicating known difficulties that occur in traditional approaches. Affect parameters are implemented, by constructing a theory that addresses emotion and is feasible in Web-learning environments.
\end{abstract}

Keywords: affect, emotions, mood, disposition, regulation, personalization, decision-making, learning.

\section{Introduction}

Web-based information systems are increasingly being used for learning and decision support applications. Computers are becoming better and more sophisticated every day. They can already perceive information related to user needs and preferences. One possible implementation of a Web-based system's interface that can appraise human needs is through the use of a set of parameters which influence the environment according to the emotional condition of the user [1]. An emotionally tense or unstable individual will be able to receive the contents of a webpage based to what he considers appropriate for his working or learning profile. A certain emotional condition demands a personalization of equivalent proportions. The user will have the capability to respond emotionally either after being asked or after a decision from the system and to inform the content presentation module about his preferences and inclinations. 
In order for a personalization system to work, it is necessary to have a solid and grounded theory and a set of personalization rules that will truly respond to user needs and change the environment to their benefit. Affective processing is a mechanism that is not fully researched and the implications from the various studies that exist in the field are often contradictory [2]. Therefore, it is of great importance to formulate a theory and especially a model of Affect and then implement a platform which takes into consideration both traditional profile as well as cognitive and affective data of the user and develop suitable system's architecture and its personalization rules.

\section{Proposed Model of Affect}

Affect is a term that includes a range of feelings that individuals experience, including discrete emotions, moods and traits such as positive and negative affectivity. There is an ongoing debate in whether emotions have a vital role in people's performance, judgement and decision making process [3] [4]. There are of course the notions of mood and disposition. The borders between the three dimensions are foggy and we cannot be certain in many occasions about the nature of the affective process. Emotions can sometimes transform into a mood and moods in a range of time can be indicative of a person's dispositional affect.

An in-depth model that grasps the complexity of these underlying concepts is the first purpose of our research. Instead of selecting one area of implementation we combine these three levels of analyses and form a typology that will help us circle effectively the affective mechanisms of the brain. In order to apply a purely psychological construct to a digital platform based on personalization rules we adjust the various theories concerning emotions having in mind to make our model flexible and applicable to users' profiles, needs and preferences. Our model has three base elements:

a) Emotional arousal is the capacity of a human being to sense and experience specific emotional situations. An effort to construct a model that predicts the role of specific emotions is beyond the scope of our research, due to the complexity and the numerous confounding variables that would make such an attempt rather impossible. We focus on arousal as an emotional mechanism and not on a number of basic emotions because it can provide some indirect measurement of general emotional mechanisms since it manages a number of emotional factors like anxiety, boredom effects, anger, tension and sadness.

b) Mood is an affective state that lasts longer than an emotion and is not as specific as an emotion can be. Moods generally have either a positive or negative valence.

c) Dispositional affect is a stable trait and tendency towards positivity or negativity. Individuals with positive affectivity tend to be cheerful and energetic and experience positive moods across a variety of situations as compared to people who tend to have low energy and be melancholic. Individuals with negative affectivity have a negative view on self and tend to be distressed and upset in relation to people who are calm and relaxed.

These basic elements that constitute the affective state of an individual play an important role in the emotionally-charged information that a person is receiving. Our model would be problematic without a regulatory mechanism of affect. For this reason we 
constructed the measure of emotion regulation that is comprised from terms like emotional intelligence, self-efficacy, emotional experience and emotional expression. Emotion regulation is the way in which an individual is perceiving and controlling his emotions. Individuals attempt to influence which emotions they have, when they have them and how they experience and express them.

\section{Incorporating Affective Factors in the Personalization Process}

In order to manipulate the parameters of our adaptive system [5] according to user characteristics, our research has to go through the stage of extracting quantified elements that represent deeper psychological and emotional abilities. The latter cannot be directly used in a web environment, but a numerical equivalent can define a personalization parameter. Our main objective is to quantify the terms of emotional arousal and emotion regulation in our first experiment and dispositional affect in the second, and see their effect on performance and the value of personalization through the aforementioned categories.

In our study, we are interested in the way that individuals process their emotions and how they interact with other elements of their information-processing system. We conducted two consecutive experiments to test a part of our theory on affection.

In the first phase of our research we examined the immediate and synchronous affective user reactions and behaviour which are covered in our model by the terms of emotional arousal and emotion regulation [6]. We hypothesized that by combining the level of arousal of an individual with the moderating role of emotion regulation, it is possible to clarify, at some extent, how affectional responses of the individual hamper or promote learning procedures. Thus, by personalizing on this concept of affect the educational content that our already developed adaptive system provides [7], we can avoid stressful instances and take full advantage of the users' cognitive capacity at any time. At a practical level, our personalization rules were based in the assumption that users with high arousal levels lacking the moderating role of emotion regulation are in a greater need of enhancing the aesthetic aspects of our system, while users with low arousal levels focus more on usability issues. Another hypothesis is that emotion regulation and arousal are negatively correlated. We propose that an individual with high emotion regulation would usually have low arousal levels because of his ability to control and organize his emotions.

In the second phase we are interested in clarifying the role of dispositional trait affect which is a global and general mood (positive or negative) and its relationship with the construct of emotion regulation. After the construction and standardization of our instruments we are currently trying to find the weighting, the importance and the implications of dispositional affect. Our hypothesis is that a user with negative affect and low regulation potential will be keener to accept and make greater use of the personalization tools that we offer him. The personalization is based on the aesthetic enhancement of the interface and on the better provision of content. The former tool aids his informational needs and the latter his processing needs. These two tools are embedded in the interface and our goal is to measure how users with specific profiles will perform with or without personalization. Participants were allocated in four categories of affection that came up from a combination of dispositional affect (positive or negative) and emotion regulation (high or low) and we assumed that users with positive affect and high regulation would perform better than users with negative and low. 
Emotional arousal and emotion regulation are concepts easily generalized, inclusive and provide some indirect measurement of general emotional mechanisms. These sub-processes manage a number of emotional factors like anxiety boredom effects, anger, feelings of self efficacy, user satisfaction etc. Among these, our current research concerning emotional arousal emphasizes on anxiety, which is probably the most indicative, while other emotional factors are to be examined within the context of a further study.

Anxiety is an unpleasant combination of emotions that includes fear, worry and uneasiness and is often accompanied by physical reactions such as high blood pressure, increased heart rate and other body signals [8] [9]. Accordingly, in order to measure emotion regulation, we are using a construct that includes the concepts of emotional intelligence, self-efficacy, emotional experience and emotional expression [10]. However, there is a considerable amount of references concerning the role of emotion and its implications on academic performance (or achievement), in terms of efficient learning [11]. Emotional intelligence seems to be an adequate predictor of the aforementioned concepts, and is a grounded enough construct, already supported by academic literature [12] [13].

Dispositional affect is a general term used more or less interchangeably with various others, such us emotion, emotionality, feeling and mood. It can be used as a label for the pleasantness-unpleasantness dimension of feeling. It can be differentiated from mood in most occasions, which is properly used for more pervasive and sustained emotional states [14]. In our research we took into consideration dispositional affect as a separate construct and we investigated its relationship with emotion regulation as well as its effect on performance. For this reason we developed a questionnaire of ten items that follows the typology of positive and negative affect. It allocates users in one of the two categories. Combined with emotion regulation they give us four categories in total that we used in our second experiment to investigate user performance and personalization efficiency.

\section{Experimental Evaluation}

\subsection{Sampling and Procedure}

All participants were students from the University of Athens. The first part of the study concerning affect was conducted with a sample of 92 students. 35\% of the participants were male and $65 \%$ were female, and their age varied from 17 to 22 with a mean age of 19 . In the second experiment 124 students participated $40 \%$ of whom were male and $60 \%$ female with exactly the same age mean and variance.

The environment in which the procedure took place was an e-learning course on algorithms; the factor of experience was controlled for. In order to evaluate the effect of matched and mismatched conditions, participants took an online assessment test on the subject they were taught (algorithms). This exam was taken as soon as the elearning procedure ended, in order to control for long-term decay effects. The dependent variable that was used to assess the effect of adaptation to users' preferences was participants' score at the online exam. 
The sample was divided in two groups: almost half of the participants were provided with information matched to their preferences, while the other half were taught in a mismatched way. We expected that users in the matched condition would outperform those in the mismatched condition.

In the first experiment, users in the matched condition with moderate and high levels of anxiety receive aesthetic enhancement of the content and navigational help and in the mismatched condition users with moderate and high levels of anxiety receive no additional help or aesthetics.

In the second experiment again half of the participants were provided with information matched to their affective preferences (aesthetic and processing facilitation), while the other half were taught in a mismatched way. Apart from the investigation on the role of personalization in general we measured performance in four categories of affection that came up from a combination of dispositional affect (positive or negative) and emotion regulation (high or low). Our hypothesis was that again (like emotional arousal) dispositional affect would be negatively correlated with emotion regulation, and that personalization tools will help users to raise their performance especially those with negative affect and low regulation skills. In this second phase apart from the aesthetic enhancement tool, participants in the negative affect category received the additional help of personalized content, because according to theory individuals with negative affect process information with a different manner (usually worse) and that is why they have extra processing needs.

\subsection{First Experiment Results}

The results of experiments conducted within the actual learning environment (table 1, 2 ), as we hypothesized, show that users with high or medium anxiety level (core and specific), lacking the moderating role of emotion regulation, are in a greater need of enhancing the aesthetic aspects of our system and the provision of additional help, in order to perform as well as low anxiety users. Users with low anxiety levels focus more on usability aspects.

Table 1. Analysis of variance between emotion regulation groups and core anxiety means

\begin{tabular}{|l|r|r|r|r|r|}
\hline & $\begin{array}{r}\text { Sum of } \\
\text { Squares }\end{array}$ & \multicolumn{1}{c|}{ df } & \multicolumn{1}{c|}{$\begin{array}{c}\text { Mean } \\
\text { Square }\end{array}$} & \multicolumn{1}{c|}{ F } & Sig. \\
\hline Between & 4.316 & 2 & 2.158 & 18.554 & .000 \\
Groups & 10.700 & 92 & .116 & & \\
Within Groups & 15.015 & 94 & & & \\
Total & & & & \\
\hline
\end{tabular}

Table 2. Analysis of variance between emotion regulation groups and specific anxiety means

\begin{tabular}{|l|r|r|r|r|r|}
\hline & $\begin{array}{r}\text { Sum of } \\
\text { Squares }\end{array}$ & \multicolumn{1}{c|}{ df } & \multicolumn{1}{c|}{$\begin{array}{c}\text { Mean } \\
\text { Square }\end{array}$} & \multicolumn{1}{c|}{ F } & Sig. \\
\hline Between & 8.345 & 2 & 4.173 & 15.226 & .000 \\
Groups & 25.213 & 92 & .274 & & \\
Within Groups & 33.558 & 94 & & & \\
Total & & & \\
\hline
\end{tabular}


Table 3. Multifactorial ANOVA (Factors - Core Anxiety, Application Specific Anxiety and Aesthetics)

\begin{tabular}{|c|c|c|c|c|c|}
\hline Source & $\begin{array}{l}\text { Type III } \\
\text { Sum of } \\
\text { Squares } \\
\end{array}$ & df & $\begin{array}{l}\text { Mean } \\
\text { Square }\end{array}$ & $F$ & Sig. \\
\hline $\begin{array}{l}\text { MatchedAesthetic } \\
\text { s } \\
\text { core_groups * } \\
\text { specific_groups * } \\
\text { MatchedAesthetic } \\
\text { s }\end{array}$ & $\begin{array}{l}\text { (a) } \\
1097.361 \\
983.259\end{array}$ & 1 & $\begin{array}{r}1097.361 \\
983.259\end{array}$ & $\begin{array}{l}4.238 \\
3.797\end{array}$ & $\begin{array}{l}.043 \\
.055\end{array}$ \\
\hline
\end{tabular}

(a) $\mathrm{R}$ Squared $=.102$ (Adjusted R Squared $=.017$ )

All types of anxiety are positively correlated with each other and negatively correlated with emotion regulation. These findings support our hypothesis and it can be argued that our theory concerning the relationship between anxiety and regulation has a logical meaning. In tables 1 and 2 an even stronger relationship between Emotion regulation and core and specific anxiety is displayed respectively. A statistically significant analysis of variance for each anxiety type shows that if we categorize the participants according to their Emotion regulation ability, then the anxiety means vary significantly with the high regulation group scoring much higher than the low one. Finally, in table 3 we can see that the two conditions (matched aesthetics/mismatched aesthetics) are differentiating the sample significantly always in relation with performance. Participants in the matched category scored higher than the ones in the mismatched one and additionally lower anxious (core or specific or both) scored higher than high anxious, always of course in relation to match/mismatch factor.

We also found that participants with low application specific anxiety perform better than participants with high specific anxiety in both matched and mismatched environments. Additionally, when a certain amount of anxiety exists, the match-mismatch factor is extremely important for user performance. Participants with matched environments scored highly while participants with mismatched environments had poor performance. Emotion regulation is negatively correlated with current anxiety. High emotion regulation means low current anxiety and low emotion regulation means high current anxiety.

Finally, current anxiety is indicative of performance, while high current anxiety is associated with test scores below average and low current anxiety with high scores. Graph 1 shows the scores that participants achieved in relation to each experimental condition.

\subsection{Second Experiment Results}

The results of the second experiment conducted again as we hypothesized, show that users with negative affect, lacking the moderating role of emotion regulation take advantage of the aesthetic aspects of our system and the provision of additional help (processing), in order to perform similarly with users with positive mood and regulation skills (see Graph 2). Additionally as it can be seen in table 4, the two notions of 
Graph 1. Mean scores (performance) in each experimental condition

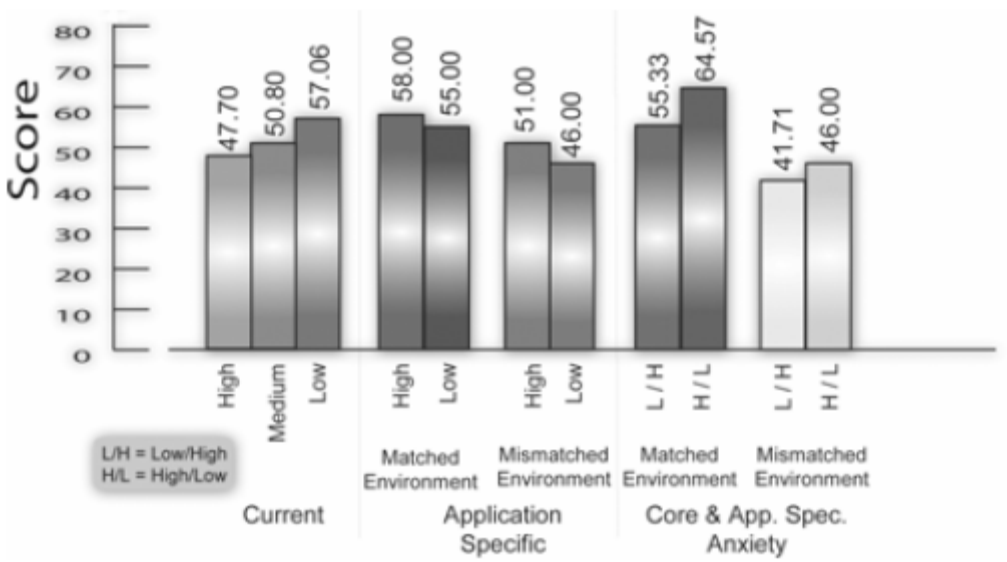

dispositional affect and emotion regulation were found to be as hypothesized significantly statistically different. A user with high regulation ability has a tendency towards positive mood and a user with low regulation ability is resilient to negative mood.

A significant finding is that the affective state of the user is having an effect on his score (Table 5 and Graph 2). Participants with positive affect perform better than participants with negative affect in both matched and mismatched environments.

Table 4. Analysis of Variance (ANOVA) between dispositional affect (positive or negative) and Emotion regulation

\section{ANOVA}

reg_means
\begin{tabular}{|l|r|r|r|r|r|}
\hline & $\begin{array}{c}\text { Sum of } \\
\text { Squares }\end{array}$ & df & Mean Square & F & Sig. \\
\hline Between Groups & 2.245 & 1 & 2.245 & 13.171 & .000 \\
Within Groups & 20.796 & 122 & .170 & & \\
Total & 23.041 & 123 & & & \\
\hline
\end{tabular}

Table 5. Analysis of Variance (ANOVA) between affective state (based on dispositional affect and regulation) and scores

\section{ANOVA}

Score \%

\begin{tabular}{|l|r|r|r|r|r|}
\hline & \multicolumn{1}{|c|}{$\begin{array}{c}\text { Sum of } \\
\text { Squares }\end{array}$} & df & Mean Square & F & Sig. \\
\hline Between Groups & 2203.378 & 3 & 734.459 & 2.699 & .049 \\
Within Groups & 32649.589 & 120 & 272.080 & & \\
Total & 34852.968 & 123 & & & \\
\hline
\end{tabular}


Graph 2. Overall scores categorized by affective type and by environment

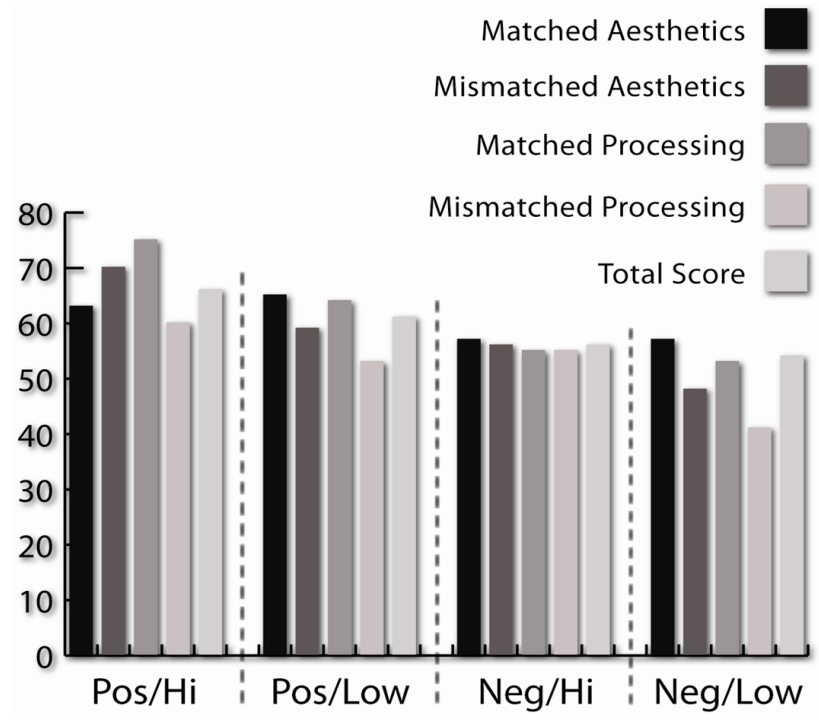

Additionally, the match-mismatch factor is extremely important for user performance. Participants with matched environments scored highly while participants with mismatched environments had poor performance.

Overall we can say that affect is greatly related to performance. Of equal importance is the notion of regulation that acts as a moderating factor to negative Affect and as a reinforcement to positive affect. The personalization techniques were proven beneficial for all users, and especially for those with negative affect. This group of users requires specific help on the interface as well as the structure and appearance of information. In our design, their informational and processing needs were met by the personalization tools of aesthetic enhancement, navigation support and content re-allocation.

\section{Conclusions}

By combining the affective state of the individual with his regulatory mechanism we can reach a conclusion of how affect infuences his learning performance. We cannot accept in advance that high emotional reactions have a negative effect on the individual since, through regulation, emotionality can be manifested as motivation and/or extra effort. Another key point in our rationale is that an affective instance cannot be described as a discrete and separate emotion but it is a more complex state in which various emotions can coexist.

Affective information can be analysed in many consecutive emotional bursts that can easily be theoretically contradictory. Various emotions and affective reactions of different (or the same) valence can exist at the same time or alternate in great speed that is difficult to grasp. Due to the complexity of the individual's affective state it is 
wise to form a typology and speak of affective types and categories and not to look for specific emotions. One possibly wrong assumption in emotion research is that discrete emotions occur in isolation. In fact, we believe that emotional reactions frequently involve more than one discrete emotion. Emotion regulation is of great importance because it can alter the outcome of the individual's behaviour from negative to positive.

We can argue that affect is greatly related to performance. Of equal importance is the notion of regulation that acts as a moderating factor to negative affect and as a reinforcement to positive affect. The personalization techniques were proven beneficial for all users, and especially for those with negative affect. This group of users requires specific help on the interface as well as the structure and appearance of information [15]. In our design, their informational and processing needs were met by the personalization tools of aesthetic enhancement, navigation support and content reallocation.

The examination of affective reactions can enrich our understanding of both personality and emotions. The issue of individual differences in affective reactions can thus be explained through the simultaneous study of user affective characteristics and learning behaviour.

\section{References}

1. Picard, R.W.: Affective Computing. MIT Press, Cambridge (1997)

2. Lewis, M., Haviland-Jones, J.M.: Handbook of emotions, 2nd edn. The Guildford Press, New York (2004)

3. Bechara, A., Damasio, H., Damasio, A.R.: Emotion, decision-making, and the orbitofrontal cortex. Cerebral Cortex 10, 295-307 (2000)

4. Levenson, R.W.: The intrapersonal functions of emotion. Cognition and Emotion 13, 481504 (1999)

5. Germanakos, P., Tsianos, N., Lekkas, Z., Mourlas, C., Samaras, G.: Capturing Essential Intrinsic User Behaviour Values for the Design of Comprehensive Web-based Personalized Environments. Computers in Human Behavior Journal, Special Issue on Integration of Human Factors in Networked Computing (2007), doi:10.1016/j.chb.2007. 07.010

6. Lekkas, Z., Tsianos, N., Germanakos, P., Mourlas, C.: Integrating Cognitive and Emotional Parameters into Designing Adaptive Hypermedia Environments. In: Proceedings of the Second European Cognitive Science Conference (EuroCogSci 2007), pp. 705-709 (2007)

7. Tsianos, N., Lekkas, Z., Germanakos, P., Mourlas, C., Samaras, G.: User-centered Profiling on the basis of Cognitive and Emotional Characteristics: An Empirical Study. In: Nejdl, W., Kay, J., Pu, P., Herder, E. (eds.) AH 2008. LNCS, vol. 5149, pp. 214-223. Springer, Heidelberg (2008)

8. Kim, J., Gorman, J.: The psychobiology of anxiety. Clinical Neuroscience Research 4, 335-347 (2005)

9. Barlow, D.H.: Anxiety and its disorders: The nature and treatment of anxiety and panic, 2nd edn. The Guilford Press, New York (2002)

10. Schunk, D.H.: Self-efficacy and cognitive skill learning. In: Ames, C., Ames, R. (eds.) Research on motivation in education. Goals and cognitions, vol. 3, pp. 13-44. Academic Press, San Diego (1989) 
11. Kort, B., Reilly, R.: Analytical Models of Emotions, Learning and Relationships: Towards an Affect-Sensitive Cognitive Machine. In: Conference on Virtual Worlds and Simulation (VWSim 2002) (2002),

http: / / affect.media.mit.edu/projectpages/lc/vworlds.pdf

12. Goleman, D.: Emotional Intelligence: why it can matter more than IQ. Bantam Books, New York (1995)

13. Salovey, P., Mayer, J.D.: Emotional intelligence. Imagination, Cognition and Personality $9,185-211(1990)$

14. Barsade, S., Brief, A., Spataro, S.: The affective revolution in organizational behavior: The emergence of a paradigm. In: Greenberg, J. (ed.) Organizational Behavior: The State of the Science, p. 352. Lawrence Erlbaum Associates, Publishers, London (2003)

15. Cassady, C.C.: The influence of cognitive test anxiety across the learning-testing cycle. Learning and Instruction 14, 569-592 (2004) 